\title{
Applications of MRI Connectomics
}

\author{
Jessica S. Damoiseaux ${ }^{1}$, Andre Altmann², Jonas Richiardi ${ }^{3}$, Sepideh Sadaghiani ${ }^{4}$ \\ Chapter to be published in: \\ Jezzard, P. \& Choi I.Y. (Eds.) Advanced Neuro Magnetic Resonance Techniques and \\ Applications. Elsevier Academic Press.
}

\begin{abstract}
${ }^{1}$ Institute of Gerontology, Department of Psychology, Wayne State University, Detroit, MI, USA. ${ }^{2}$ Centre for Medical Image Computing (CMIC), Department of Medical Physics and Biomedical Engineering, University College London (UCL), London, United Kingdom.

${ }^{3}$ Department of Radiology, Lausanne University Hospital and University of Lausanne, Switzerland.

${ }^{4}$ Psychology Department, Beckman Institute for Advanced Science and Technology, University of Illinois at Urbana-Champaign, Urbana, IL, USA.
\end{abstract}

\begin{abstract}
:
Structural and functional brain connectomics are considered a basis for an individual's behavior and cognition. Therefore, deviations from typical connectivity patterns may indicate disease processes, and can potentially serve as disease biomarkers. To date, the direct clinical application of brain connectivity measures for diagnostics or treatment is limited. Nonetheless, the extant literature on fundamental and clinical research applications reveals important advances in our understanding of typical and atypical brain structure and function. In this chapter we discuss the current status of the field regarding: (1) the impact of the connectome on cognitive processes and behavior, (2) the connectome across the lifespan, and (3) clinical research applications of connectomics. In addition, we highlight some limitations of connectomics for research and clinical translation.
\end{abstract}

Keywords: connectomics, functional connectivity; structural connectivity; MRI; cognition; aging; clinical applications. 


\section{Introduction}

How can magnetic resonance imaging (MRI) connectomics be applied to study typical brain structure and function, and be used in a clinical setting to inform diagnosis, prognosis, or treatment of patients? In the current chapter we will discuss possible applications of MRI Connectomics as assessed with functional and structural connectivity. The previous two chapters in "Part 4" of this book detail multiple techniques to assess functional and structural (diffusion-based) brain connectivity. In short, functional connectivity is a measure of the association of functional MRI (fMRI) derived Blood Oxygenation Level Dependent (BOLD) fluctuations across brain regions, commonly expressed as correlation coefficients. As BOLD is an indirect measure of neuronal function, functional connectivity is considered to reflect systemlevel neuronal signaling between brain regions. Brain regions with higher measures of functional connectivity are interpreted as having more synchronous neuronal signaling, and therefore as more likely belonging to the same neural network. The extant literature has identified about 7-10 canonical intrinsic connectivity networks (ICNs) including sensory, motor and higher cognitive systems (cf. chapter 12 on functional connectivity). When comparing functional connectivity measures within and between individuals, e.g., based on features such as behavior, age, physical health etc., it is tempting to assume higher connectivity indicates better brain function. However, the interpretation of connectivity differences is not as straightforward; depending on the specific brain regions involved, and other circumstances, lower connectivity can also be considered advantageous.

Structural connectivity, as described in this book, is derived from diffusion weighted imaging (DWI). Structural connectivity "strength" can be assessed in multiple ways, such as an average diffusion measure per tract of interest (e.g., fractional anisotropy, mean diffusivity, neurite density etc.), or as the number of streamlines between brain regions of interest using probabilistic tractography. Diffusion-derived structural connectivity measures are considered to reflect white matter pathways (i.e., the direction of axons). Brain regions with higher structural connectivity are presumed to have more dense white matter tracts and/or higher white matter integrity, and therefore more intact or stronger anatomical connections. This interpretation is not without controversy, though, as discussed by (Jones et al., 2013), and careful consideration of the actual measures extracted is warranted when interpreting the results.

A common approach to examine whole brain or system-level connectomics is the use of brain graphs (see Box 14.1 for a brief primer on brain graph analyses). A brain graph approach allows us to determine specific network properties and can be applied similarly to connectivity measures extracted from multiple imaging modalities. Therefore, in both the functional and structural connectomics literature similar network properties are discussed.

Connectivity signatures are heritable to some degree and therefore represent a conserved biological property rather than a mere temporary snapshot of brain activity. Connectivity patterns are expected to relate to cognitive function, and deviations from normative connectivity patterns can be an indicator of disease processes and consequently serve as disease biomarkers. There is currently limited direct clinical application of brain connectivity measures for diagnostics or treatment. Nevertheless, there are multiple fundamental and clinical research applications that increase our understanding of typical and atypical brain structure and function. In the following sections we will provide examples of our current understanding of (1) the impact 
of the connectome on cognitive processes and behavior, (2) the connectome across the lifespan, and (3) clinical research applications of connectomics.

\section{Impact of the connectome on cognitive processes and behavior.}

Ultimately, the functional significance of the connectome lies in its role in healthy cognition, and by extension, in cognitive consequences of connectome aberrations. Associations with cognitive abilities and behavioral outcomes are further important for demonstrating that neuroimagingderived structural and functional connectomes reflect genuine neural connectivity rather than noise (cf. discussion of methodological limitations in section 5). Several general approaches have been taken to establish and describe such impact on cognitive processes and behavior (Figure 1). Specifically, differences in behavioral measures have been associated with differences in the connectome across individuals (section 2.1.) or, alternatively, within individuals (2.2). In both of these approaches, behavioral associations have been explored for the DWI-derived structural connectome, the fMRI-derived "static" (i.e., time-averaged) functional connectome, and the fMRI-derived "dynamic" (i.e., time-varying) reconfigurations of the functional connectome (cf. chapter 12 for definitions of static and dynamic connectivity). In the following, we will discuss examples of each of these approaches.

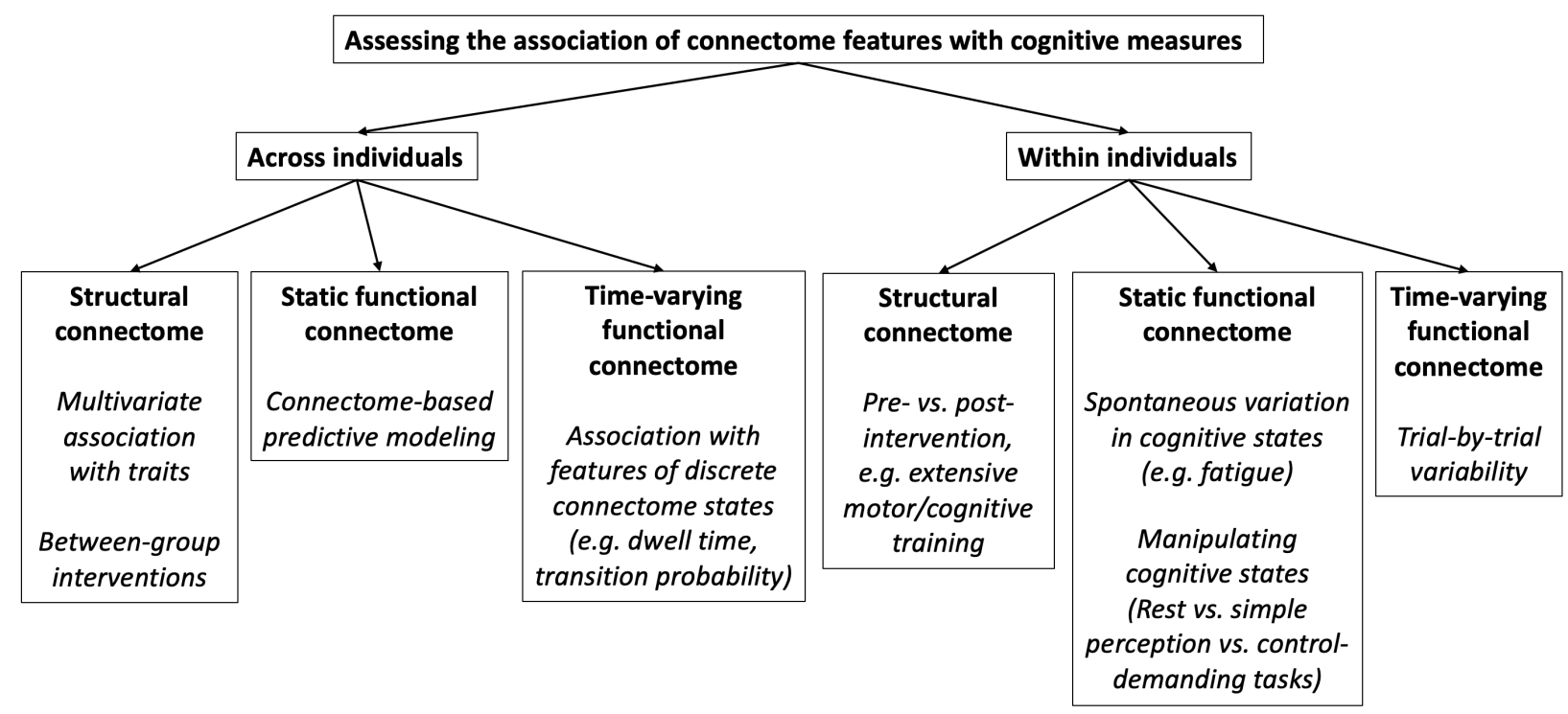

Figure 1: Overview of recent research approaches to characterize the association of connectome features with cognitive and behavioral processes. The approaches range from between-to within-group designs and from association to intervention studies. The highlighted approaches are not exhaustive but provide examples from studies that have proven informative.

\subsection{Association of connectome features with cognitive abilities across subjects.}

An important avenue for understanding the cognitive relevance of the connectome is to study how cognition and connectome features co-vary across individuals. For inter-individual comparisons, cognitive abilities are commonly assessed by self-report questionnaires or by 
performance in standardized neuropsychological tests or other cognitive tasks. The following examples explore which specific structural and functional connectome features are behaviorally relevant. This section focuses on inter-individual studies of relatively homogenous populations; for age- and disease-related differences cf. the sections 3 and 4 of this chapter, respectively.

\section{Structural connectome across individuals.}

Structural connectivity reflects the cumulative effect of genes combined with the impact of the environment, the latter including life-long experience and learning. This representation extends from individual synapses to the macroscale connectome observable with DWI. Consequently, it is expected that specific features of the DWI-derived structural connectome are linked to individuals' cognition. While the relationship of targeted single connections to specific cognitive abilities has long been studied, recent imaging and analysis advances have enabled association studies of the distributed structural connectome at once. Specifically, cognitive associations have been assessed concurrently for all edges or nodes of the DWI-derived connectome using multivariate analyses, and for global topological measures using network science (see Box 14.1). By applying these approaches, associations with higher-order control and executive functions were identified. These associations involve (often sparse sets of) structural connections widely distributed across anatomical space, rather than being e.g., confined to connectivity within specific canonical ICNs.

\section{Phenotyping individuals based on their static functional connectome.}

All humans share a common level of spatial organization of the static functional connectome, most notably the presence of the previously mentioned set of so-called canonical ICNs or resting state networks each comprising a specific group of distributed brain regions (cf. chapter 12). However, the exact instantiation of this spatial organization differs across individuals in characteristic ways similar to an individual's fingerprint. The characteristic differences, distributed throughout the brain, can be used to identify an individual from others. Given the success of such identification or functional connectome "fingerprinting", one might expect that individuals' cognitive abilities could be predicted from relevant connectome features (e.g. using connectome-based predictive modelling (Shen et al., 2017). Indeed, despite the close dependence of functional connectivity on anatomy (the way traffic patterns depend on roads), certain functional connectome features are uniquely associated with cognitive abilities beyond structural connectivity. Such features from resting state fMRI are associated with a person's cognitive abilities such as fluid intelligence (the ability of abstract reasoning and problemsolving), sustained attention, and performance on perception tasks.

It should be noted that while studies into inter-individual differences most commonly use fMRI data from task-free resting state, individuals' characteristic functional connectome features are largely stable within individuals irrespective of cognitive state. Further, the functional connectome's prediction of cognitive traits may improve when fMRI is acquired during targeted cognitive states that enhance relevant connectome idiosyncrasies. For example, the level of global integration of the functional connectome (i.e., the extent to which densely connected clusters of are interconnected; cf. Box 14.1) during a demanding working memory and cognitive control task is associated with individuals' task performance (Shine et al., 2016). 
Time-varying dynamics of the functional connectome as traits.

Many everyday cognitive processes, such as task-switching and multitasking, require cognitive flexibility. It has been suggested that time-varying changes in the functional connectome permit new alliances to form and dissolve between brain regions, described as "connectome flexibility". These different configurations of the functional connectome are suggested to constitute different "cognitive architectures", each supporting a different type of cognitive process. In line with this view, one approach to functional connectome dynamics is to define multiple, relatively discrete connectome states and characterize how their spatial, topological, and temporal features affect cognition. For example, individuals with more frequent occurrence of a certain connectome state (characterized by high variability of connectivity strength) during resting state perform better in common measures of task-switching and working memory (Nomi et al., 2017). It should be noted that due to technical limitations (see section 5) fMRI typically captures functional connectome dynamics only on the temporal order of tens of seconds. Many cognitively relevant connectivity changes therefore will require methods other than MRI.

\subsection{Association of connectome features with cognitive states within subjects}

Beyond comparing individuals with each other, a second window into the cognitive significance of connectome features is to study how such features co-vary with changes in behavior within an individual. This approach commonly takes advantage of spontaneous variability of behavior and cognitive performance or, alternatively, changes in such functions due to interventions and training. Changes in large-scale structural connectivity can be observed upon relatively longterm and extensive learning. Conversely, static functional connectivity can additionally capture more rapid reorganization e.g., upon switching cognitive states (e.g. across resting state and different task paradigms). Finally, even within a task paradigm, trial-to-trial variability in behavior can be linked to dynamic changes in the functional connectome. In the following, we explore examples of associations of structural, static functional, and dynamic functional connectome features with cognition and behavior within individuals.

\section{Structural connectome and learning.}

A straightforward intervention to study the behavioral impact of structural connectome changes within an individual is to induce learning. For example, two months of working memory training enhances efficiency of information flow (a network science measure, cf. Box 14.1) in a structural network of frontal and parietal nodes (Caeyenberghs et al., 2016). Importantly, subjects in the training group (but not a control group) showed cognitive transfer in terms of improved performance on other tasks involving working memory post-training compared to pre-training.

Static functional connectome and cognitive states.

While the spatial organization of the functional connectome is extremely stable over cognitive contexts, the subtle changes that occur between such contexts can be meaningfully linked to cognitive processes. A robust example of such subtle changes is the level of global topological integration (see Box 14.1) increasing gradually with the level of cognitive demands from resting state and simple motor tasks to cognitive control-demanding paradigms. Here, the relevance to cognition is either assessed by a between-subject association with performance (see section 2.1., e.g., (Shine et al., 2016), or indirectly inferred from the expectation that cognitively 
demanding tasks require information exchange across an especially large number of regions, and thus would benefit from topological integration. In addition to experimentally controlled changes in cognitive/mental state, spontaneous fluctuations e.g., of levels of vigilance and fatigue have been associated with subtle changes in the functional connectome.

Time-varying dynamics of the functional connectome and behavioral variability. Cognitive processes are often highly variable within an individual even when stimuli and external conditions are held constant. The association of this variability with changes in the momentary state of the connectome sheds light into the cognitive significance of the latter. As discussed above, analyzing task-based fMRI data in a continuous manner similar to resting state approaches (e.g., (Shine et al., 2016) provides important information about taskdependent functional connectivity. However, intrinsically driven changes in the functional connectome may remain non-dissociable from task-evoked co-activation. To dissociate the two and understand how the former affects the latter, connectivity has been studied during prestimulus time periods. These studies use stimuli that are identical or directly comparable from trial to trial, yet cause different behavioral outcomes each time, such as threshold-level or ambiguous stimuli. For example, a machine learning approach (cf. Box 14.1) applied to prestimulus functional connectivity can predict (with above-chance accuracy) the participant's success or failure to detect a faint sound on a given trial (Sadaghiani et al., 2015). Further, the subject is more likely to hear the sound when the functional communities are more segregated (measured as high modularity, cf. Box 14.1) just prior to the sound. It is likely that the connectome switches between more globally segregated states optimal for simple perception or motor output on the one hand, and more globally integrated states for cognitive controldemanding tasks on the other. Together with investigations of continuous task periods and taskfree resting state as discussed in the previous sections, evidence is converging for the cognitive importance of global topological segregation/integration of functional connectome states.

\section{The connectome across the lifespan}

Even though general brain network organization is largely consistent across individuals, as discussed above, differences between individuals have been observed. One important modifier of brain connectomics, which affects us all, is an individual's age. The observed age-related differences across the lifespan suggest that the connectome undergoes structural and functional changes during development and aging.

\subsection{Age-related within and between network connectivity changes.}

Based on decades of research, we know that our cognitive abilities change with age. Typically, the trajectory of age-related cognitive changes shows an improvement during development, which peaks in early adulthood, and then gradually declines with older age. A similar pattern of developmental maturation followed by gradual age-related decline is observed in the brain connectome (see Figure 2). A particularly consistent observation, mostly from cross-sectional studies, indicates that functional connectivity within the so-called default mode network, one of the canonical ICNs, increases with development and declines throughout older adulthood. The default mode network consists of some of the brain's main cortical hubs, such as the posterior cingulate cortex and medial prefrontal cortex, and is generally involved in self-referential 
processes, emotional processes, and memory function. It plays a central role in supporting our cognitive abilities, and its age-related connectivity changes are reflected in the observed cognitive changes. The extant literature on structural connectivity reveals a similar pattern, where rich club organization (encompassing brain regions that overlap with the default mode network) shows an age-related decline. Most studies that examined functional and structural connectivity in the same sample reveal that these measures are closely aligned (although functional connectivity can exist between brain regions in the absence of direct structural connectivity), and that they are similarly affected by aging. See (Damoiseaux, 2017) for a review on this topic.

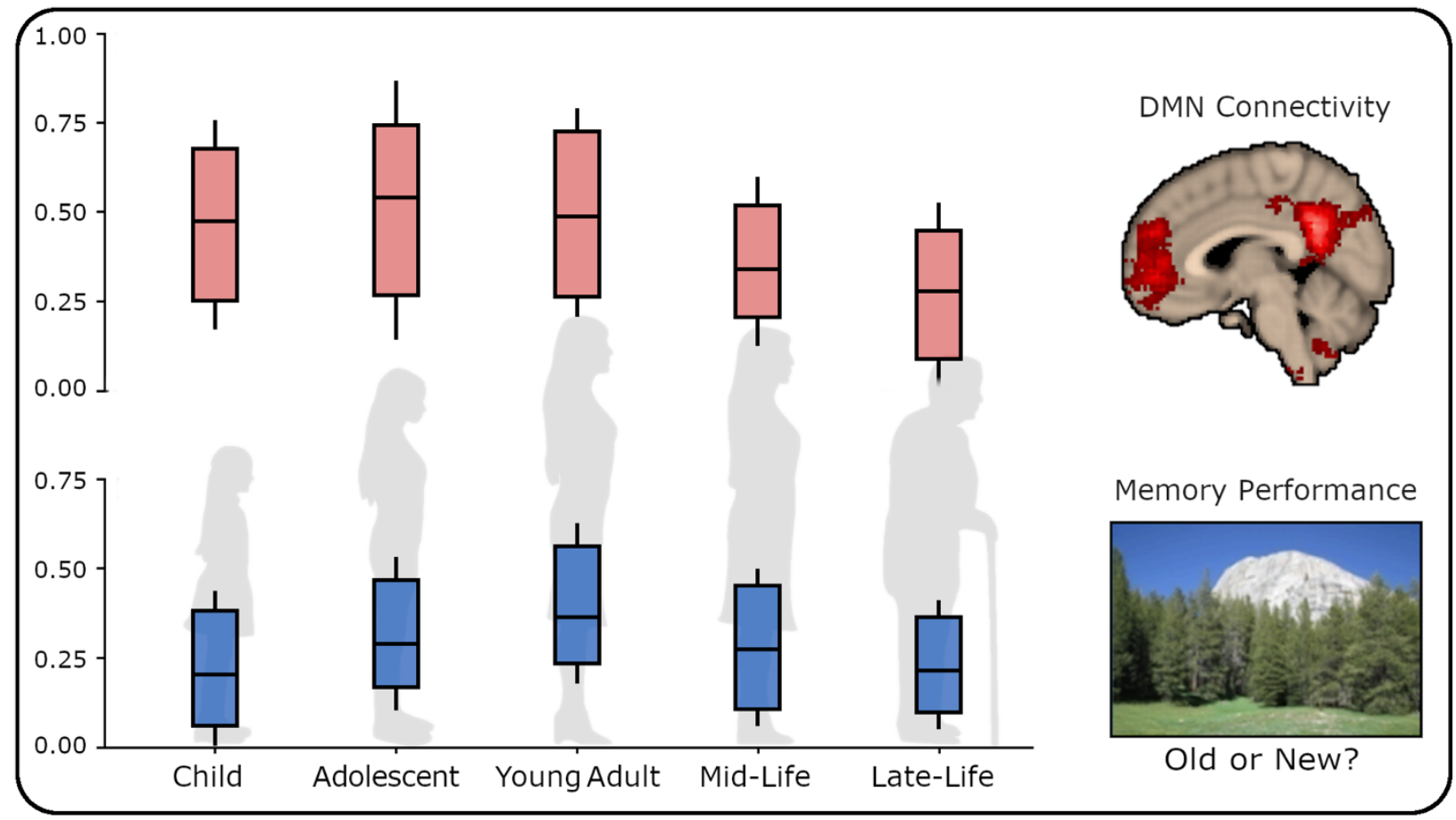

Figure 2: Illustration of the differences in connectomics and memory performance across the lifespan. Default mode network (DMN) functional connectivity (top) and memory performance (retrieval accuracy) for scenes (bottom) both show developmental maturation, peak during young adulthood, and gradually decline during mid-life and older adulthood. Based on unpublished data.

In addition to the specific changes in the default mode network, the brain's global network organization also changes across the lifespan. Several studies indicate that across the adult lifespan within-network connectivity decreases while between network connectivity increases. These brain changes indicate neural network dedifferentiation, meaning a reduction in functional network specialization with older age. Such network dedifferentiation implies less optimal brain function, possibly reflecting a compensation mechanism to maintain cognitive performance. To do so, additional brain regions are recruited to compensate for signal loss within specialized networks. However, it could also reflect noisy signal propagation due to age-related pathology that in turn leads to a reduction of neural selectivity. Either way, the observation of age-related differences in MRI derived brain network measures provides us with an understanding of typical brain aging across the lifespan. 
It has to be noted that our current understanding of age-related changes is mostly based on cross-sectional studies. Some of the relatively few longitudinal studies done appear to align with the cross-sectional findings, however, others show no change over time. More longitudinal studies, spanning longer periods of time, are needed to assess the actual trajectory of agerelated brain changes.

\subsection{Typical brain aging informs identification of pathological brain aging.}

As outlined above, the existing literature on age-related connectivity changes provides evidence in support of the applicability of connectomics to reveal individual differences in brain structure and function. One potential application of connectomics as a biomarker for an individual's brain health is the "brain-age" approach (Cole and Franke, 2017). In this approach an individual's biological brain age can be estimated based on their brain network measures and compared to their chronological age. If, for example, an adults' brain age is higher than their chronological age, this indicates accelerated aging, which in turn may indicate potential neurodegenerative disease. Our knowledge of typical brain aging can thus inform the identification of possible pathological brain processes. How connectomics may aid in the diagnosis, prognosis and treatment of clinical disorders will be discussed in the next section.

\section{Clinical research applications of connectomics}

Analyzing brain connectivity is mainly still an academic research tool to better understand brain function and organization. However, since the very beginning of connectome-based research, researchers have recognized the potential of connectivity-based measures in clinical settings. The following sections demonstrate examples of connectomics applications to aid diagnosis, disease prognosis and clinical intervention.

\subsection{Connectomics reflects biology and therefore probably disease pathways}

Both structural and functional connectivity exhibit a substantial degree of heritability, i.e., a person's genetic markup controls features of the connectome. Likewise, many disorders of the nervous system have been linked to genetic risk factors. One pertinent question is whether disease associated genetic risk variants exercise their effect on features of the connectome in healthy individuals, which would afford a better characterization of people at an increased disease risk. Indeed, in Alzheimer's disease (AD), the most common form of dementia, the $\varepsilon 4$ allele of the apolipoprotein $E(A P O E)$, the strongest common risk factor for $A D$, showed altered connectivity with regions participating in the default mode network in healthy adults across various studies (e.g., (Damoiseaux et al., 2012); Figure 3). Similarly, in a cohort of typically developing children, carriers of a risk variant the autism risk gene CNTNAP2 were found to have altered connectivity with the medial prefrontal cortex (Figure 3; (Scott-Van Zeeland et al., 2010). A different approach, used in schizophrenia research, found that increased polygenic risk (a score incorporating many hundreds of possibly weak genetic effects) was associated with reduced connectivity in a cluster primarily encompassing the visual, default mode, and frontoparietal systems (Cao et al., 2020). 
Taken together across various studies, it seems that healthy people who carry genetic variants that increase their risk for developing a brain disorder exhibit changes in their structural and/or functional connectome that precede clinical symptoms.

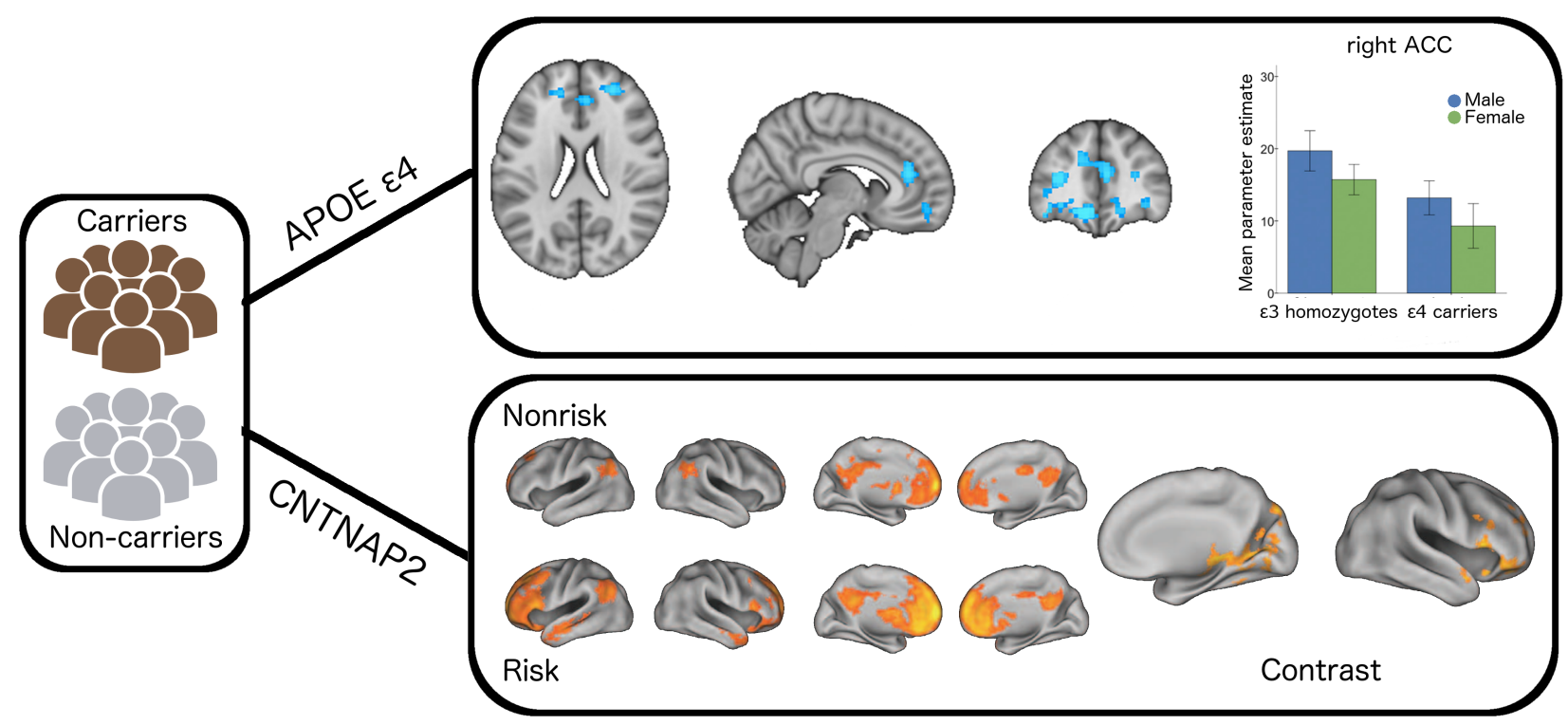

Figure 3: Genetic effects on functional connectivity. Healthy older adults carrying the APOE $\varepsilon 4$ allele show decreased functional connectivity in the anterior default mode network (top panel). Adapted from (Damoiseaux et al., 2012) with permission from the author. Functional connectivity with the medial prefrontal cortex is associated with CNTNAP2: non-risk subjects show relatively greater long-range anterior-posterior connectivity than risk carriers as well as relatively greater right frontal connectivity in risk carriers compared to the non-risk group (bottom panel). Adapted from (Scott-Van Zeeland et al., 2010) with permission from the American Association for the Advancement of Science.

\subsection{Connectomics as tool for (differential) diagnosis}

There is evidence from numerous diseases (neurodegenerative, autoimmune, psychiatric, and others) that there are differences in connectomics between patient and control groups comparable to differences across subjects with varying levels of cognitive abilities (cf. section 2.1). Thus, a strand of research has focused on eliciting sensitive and specific connectomicsderived quantities that can be used as diagnosis biomarkers. These endophenotypes of the disease typically do not rely on symptomatology, but for many of them good statistical relationships are shown with established diagnostic criteria or clinical scales. For some diseases, such as multiple sclerosis (MS), there has been a frustrating lack of correlation between imaging semiology and clinical scales, a phenomenon known as the "clinicoradiological paradox". One tenet of proponents of connectomics-derived biomarkers is that these biomarkers could pick up more subtle disease associations and help bridge this gap. White matter lesions in MS cause demyelination and subsequent axonal transection, thereby causing important alterations in white matter connectivity. There is a well-known spatial distribution preference for white matter lesions, in particular around the ventricles, meaning that specific tracts such as the cortico-spinal tract are often impacted. Such white matter lesions impact not only the structural connectome, but also the large-scale organization of the functional connectome, which displays decreases in the network science measures (Box 14.1) of 
efficiency (Liu et al., 2017) and small-worldness (Faivre et al., 2016). These large-scale changes can be used as biomarkers enabling diagnosis of MS patients, although the specificity has not been fully established for use in a clinical context, in particular for differential diagnosis.

A challenging clinical problem is the differentiation between distinct but related clinical syndromes that share a range of symptoms. Imaging biomarkers, including structural and functional connectivity, are being explored to assist with differential diagnosis. In an early work, Seeley et al. (2009) reported that different neurodegenerative disorders exhibit characteristic patterns of gray matter loss that spatially overlap with canonical ICNs. For instance, AD imaging signatures tend to appear first in the default mode network, whereas frontotemporal dementia (FTD) imaging signatures are first located in the so-called salience ICN. Connectivity-based biomarkers are investigated to better understand and discriminate between subtypes of syndromes. In post-traumatic stress disorder (PTSD), for example, functional connectivity between the periaqueductal gray and regions associated with emotion processing was increased in patients compared to controls. However, only in patients with the dissociative PTSD subtype there was also increased connectivity between the ventrolateral periaqueductal gray and brain regions known to be involved in passive coping strategies (Harricharan et al., 2016).

\subsection{Connectomics for prognosis and relationship with clinical scales}

Typically, prognosis for brain disease is established on a combination of demographic factors, comorbidities, neurological findings including specialized clinical scales, radiological findings (mostly based on structural imaging with various contrasts), and laboratory tests. With connectomics the relationships between brain regions and large-scale changes in connectomics caused by the disease, can be leveraged to offer new perspectives on the possible future evolution of the patient's brain reorganization through adaptive or maladaptive plasticity. For example, in stroke there is evidence that ischemic lesions cause changes in both structural and functional connectomes, leading to disconnection and reconfiguration of brain networks distal to the lesion (Carrera and Tononi, 2014). Observing brain networks that are either still intact, impacted by stroke, or at future risk can have important implications for the rehabilitation potential of specific patients and guiding therapy. Indeed, it has been reported that larger topology changes were associated with larger residual symptom burden several months after the acute phase (Schlemm et al., 2020).

Another example is FTD, which has been characterized by frontolimbic connectivity disruption leading to unconstrained prefrontal connectivity. Research showed that in patients with the behavioral variant or semantic dementia, the extent of prefrontal hyperconnectivity and frontolimbic disconnection reflected apathy and disinhibition scores, respectively (Farb et al., 2013).

\subsection{Connectomics for treatment planning and response prediction}

Choosing treatment options is based on assumptions about the patient's response to a specific treatment course. This can for example be a choice between either drug treatment or surgical intervention, several types of psychological therapy, or several types of drugs available for a particular condition. There has been increasing research about the use of connectomics to 
guide treatment choice, sometimes focusing on the connectivity between very specific brain regions, with several promising results in neuropsychiatric disorders (as illustrated in Figure 4). For example, in depression, several studies aimed to predict the response to electro-convulsive therapy based on functional connectivity. These studies show that a decrease in depressive symptoms after electro-convulsive therapy was correlated with pre-intervention connectivity of the subcallosal cingulate cortex (Argyelan et al., 2016).

Another example is Parkinson's disease, for which deep brain stimulation (DBS) is an established treatment option. However, clinical response is highly variable and may depend on the connectivity profile of the simulated brain region. (Horn et al., 2017) identified the volume of tissue activated by DBS in a given patient and combined this information with group-level structural connectome to identify affected fiber tracts. Moreover, they used the volume of tissue activated as a seed in a large cohort to establish the functional connectivity profile. Using these group level structural and functional connectomes improved the prediction of motor scores (as measured with the Unified Parkinson Disease Rating Scale-III) in response to DBS treatment, and may be used to revise or guide DBS.

Brain surgery may be needed to treat refractory epilepsy and brain tumors. Detailed planning is required to avoid damage to critical brain regions (e.g., those involved in memory, language and motor functions) and to maintain acceptable quality of life post-surgery. Traditionally, a personalized mapping of relevant brain regions, such as the eloquent cortex, is conducted using task-based $\mathrm{fMRI}$ or intraoperative electrocortical stimulation. Recent advances use functional connectivity to better predict treatment outcomes and to fine-map critical cortical regions (Sair et al., 2016).
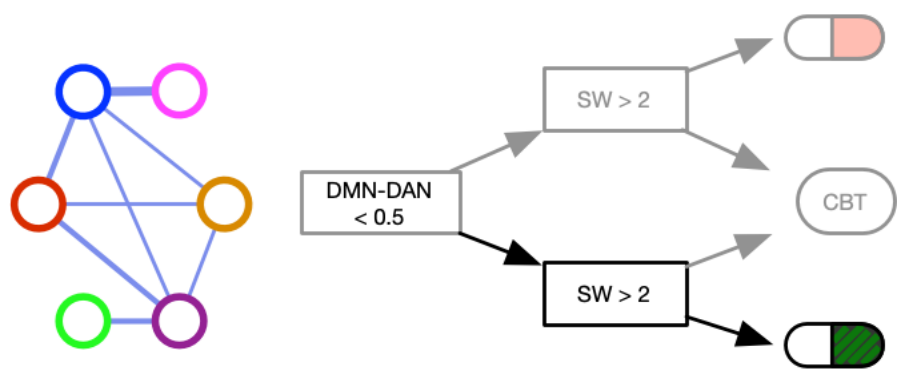

Patient A
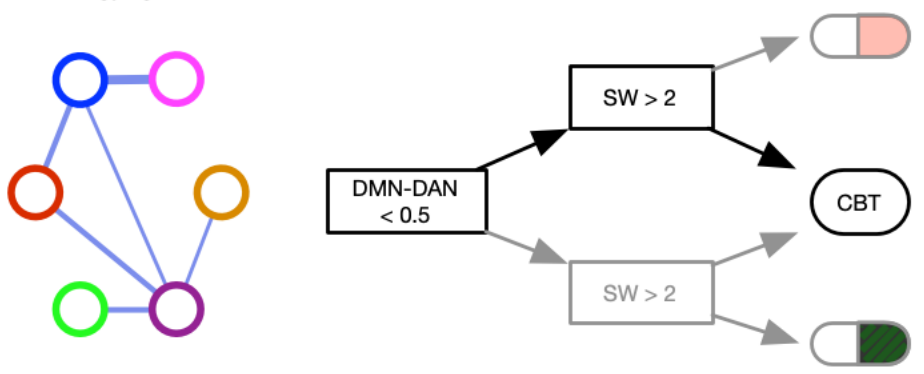

Patient B

Figure 4: Illustration of the use of connectomics for treatment planning in psychiatric disorders. Based on strength of connections between certain ICNs, or specific network science metrics (see Box 14.1), each patient can be oriented towards the most appropriate pharmacological (striped green pill or filled red pill) or psychological treatment (CBT, cognitive behavioral therapy). DMN: default-mode network. DAN: attention network. SW: Small world index. 


\section{Limitations for research and clinical translation}

Given all the promising results we have mentioned in this chapter thus far, one may wonder where are these tools used in actual clinical settings? There are in fact a number of obstacles in bringing these results to use, some of which are shared by other modalities such as structural MRI. Here, we focus on those issues that are more specifically preventing MRI connectomics from being used clinically, and that also pose problems for non-clinical research.

A perennial problem for fMRI-based connectomics, but also DWI-based connectomics, is head motion. Connectivity is modulated by motion in a spatially-dependent fashion, with differing effects in long- and short-range estimates. This is particularly problematic when two groups are compared, such as patients and controls, as group differences in motion alone can cause considerable group differences in connectivity patterns, affecting all analysis methods outlined in Box 14.1. Possible mitigation strategies include prospective motion correction, embedded cameras for head motion tracking, or a posteriori motion compensation, including independent component analysis (ICA)-based approaches such as ICA-FIX or motion "scrubbing" for fMRI. Other unwanted physiological signals such as breathing or heart rate variability can also impact the BOLD signal, in large part due to the low sampling frequency which causes aliasing into the frequency bands of interest for resting-state analysis. Mitigation strategies include low TR acquisition, or retrospective correction using various algorithms (Caballero-Gaudes and Reynolds, 2017).

There are additional confounds for functional connectivity concerning the cognitive states of the subjects. Among those, sleep (practically the loss of consciousness) imposes a drastic reorganization of the connectome such as the breakdown of thalamo-cortical coupling (as illustrated in Figure 5 by the functional connectivity difference between subcortical regions (SC) and the rest of the brain). Some diseases or medications may affect the participants' vigilance and render them more prone to doze off during the MRI session. Seemingly harmless events such as enjoying a cup of coffee may alter functional connectivity. The sensitivity of the functional connectome to pathological emotional states (such as anxiety disorders) renders it susceptible to the 'normal' range of emotional states and affects its utility as a biomarker. Lastly, the seemingly simple instruction to "lie still in the MRI scanner and to not think of anything in particular" may be difficult to follow for some patient groups (e.g., AD, ADHD, PD).

A further complication is that connectomics measures are modulated by demographic factors such as age (see section 3), sex and genetic ancestry. Some of these factors, like sex and genetic ancestry, are known to exercise a strong influence on measures such as intra cranial volume and regional brain morphology. It is still subject of ongoing research to properly adjust for these factors in connectivity analyses. However, functional connectivity may be more subtly influenced by differences such as the native language or bilingualism (Ge et al., 2015).

One of the main issues in bringing $\mathrm{fMRI}$ and high-quality DWI to the clinic is acquisition time. While around 5 minutes is sufficient to acquire a high resolution T1-weighted MRI which is used for almost all brain diseases, adding fMRI acquisition will typically add 6-7 minutes at the 
minimum, and adding DWI of sufficient quality to permit tractography (e.g. multishell imaging) will add at least 7 minutes of scan time. While these acquisition times are steadily decreasing with the widespread adoption of techniques like compressed sensing, multiband imaging, or deep learning-based reconstruction, $\mathrm{FMRI}$ and DWI must compete for acquisition time with other well-established sequences such as FLAIR or perfusion imaging, which have clearly demonstrated their clinical utility. For radiology departments, adding more sequences means decreasing patient throughput, at a time where clinical imaging exams are more and more requested by other medical specialties.

Finally, an important issue is that $\mathrm{fMRI}$ and DWI data acquired on different machines (in multiple sites) can differ very importantly in terms of technical parameters such as spatial and temporal resolution, contrast, number and distribution of diffusion directions, diffusion b-values, etc. These differences will lead to vastly different connectomics, even for the same patient. Two trends offer hope that site differences can be overcome: 1) several initiatives, notably in the radiomics field, are attempting to standardize specific biomarkers; 2) post-acquisition harmonization is progressing rapidly, driven by progress in deep learning (Tax et al., 2019).
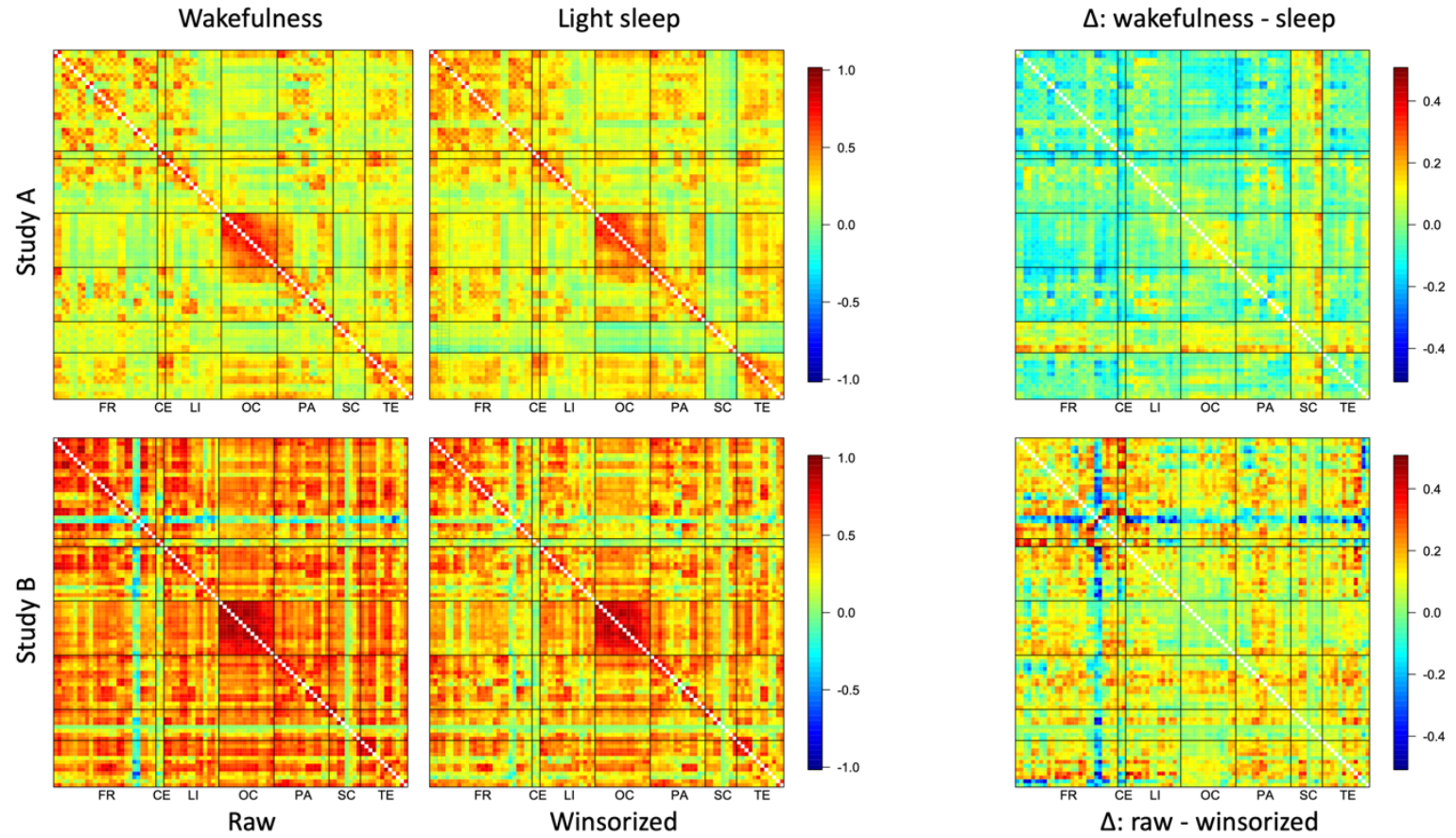

Figure 5: Effect of vigilance states and movement artifacts on functional connectivity. Matrices display regional connectivity using Spearman correlation (left and middle columns) or their difference (right column). Top row: Transitioning from wakefulness to light sleep reduces thalamo-cortical connectivity (as part of the SC regions) with group difference showing an absolute change in correlation of around 0.3; based on data from (Spoormaker et al., 2010). Bottom row: Movement can lead to widespread artificially increased connectivity (about 0.4), which can be reduced by using robust connectivity estimates. FR=Frontal; $C E=C e n t r a l ; ~ L i=L i m b i c ; O C=O c c i p i t a l ; P A=P a r i e t a l ;$ $\mathrm{SC}=$ subcortical; TE=Temporal lobe.2 


\section{Concluding remarks}

Over the last decade, MRI-derived connectivity investigations have made rapid progress towards studying the whole-brain connectome simultaneously and harnessing its multivariate information using mathematical tools such as machine learning and network science. This progress has been enabled by advances in imaging technology and, more importantly, the discovery of the intrinsic nature of connectome organization especially in functional MRI. Connectome approaches have revealed associations with cognitive processes, development and aging, genetic makeup and neurological and psychiatric disorders. These associations have not yet been harnessed for clinical diagnosis, prognosis, and treatment response prediction. Such translational impact is expected to become attainable as studies advance from the group level to characterizing functionally consequential individual differences. Both basic brain research and precision medicine are likely to benefit strongly from this development over the next decade.

\section{Box 14.1: ANALYSIS AND INFERENCE WITH BRAIN GRAPHS}

Once they are constructed, brain graphs can be analyzed at the whole-graph level, subgraph level, vertex level, or connection level, depending on the goal of the analysis. Mathematical tools to analyze and model brain graphs can be roughly grouped into four fuzzy categories: statistical approaches, machine learning approaches, network science approaches, and graph signal processing approaches (see Figure 6). In statistical approaches, the goal is to perform hypothesis testing on groups of graphs, subgraphs, vertices or edges. Several techniques developed specifically for graphs, exploit subgraphs to gain power, such as the networkbased statistics (Zalesky et al., 2010), or two-step (screening-filtering) testing (Meskaldji et al., 2015). Machine learning approaches focus instead on prediction using individual graphs, making them particularly suitable for clinical applications. There has been tremendous progress in graph-based machine learning in the last few years, especially in representation learning, either with graph embeddings (representing a graph as a vector) or with graph neural networks (end-to-end learning). While these approaches typically have very good predictive ability, they can be difficult to interpret in terms of specific brain regions or their interactions. Network science approaches, instead, propose various graph topological metrics that can be readily interpreted to yield neuroscientific insights. Notably, the notions of modularity (representing integration versus segregation of function), hubs (representing parts of the brain which play a central role in information exchange) or efficiency can be expressed and estimated compactly. These network metrics help place analysis results within a systemsoriented frame of reference; however, readers should be cautious because mathematical definitions often differ between authors. Lastly, graph signal processing approaches have gained traction recently in the neuroimaging community, and propose to translate common concepts in signal processing, such as filtering, downsampling, or Fourier analysis to the domain of brain graphs. 


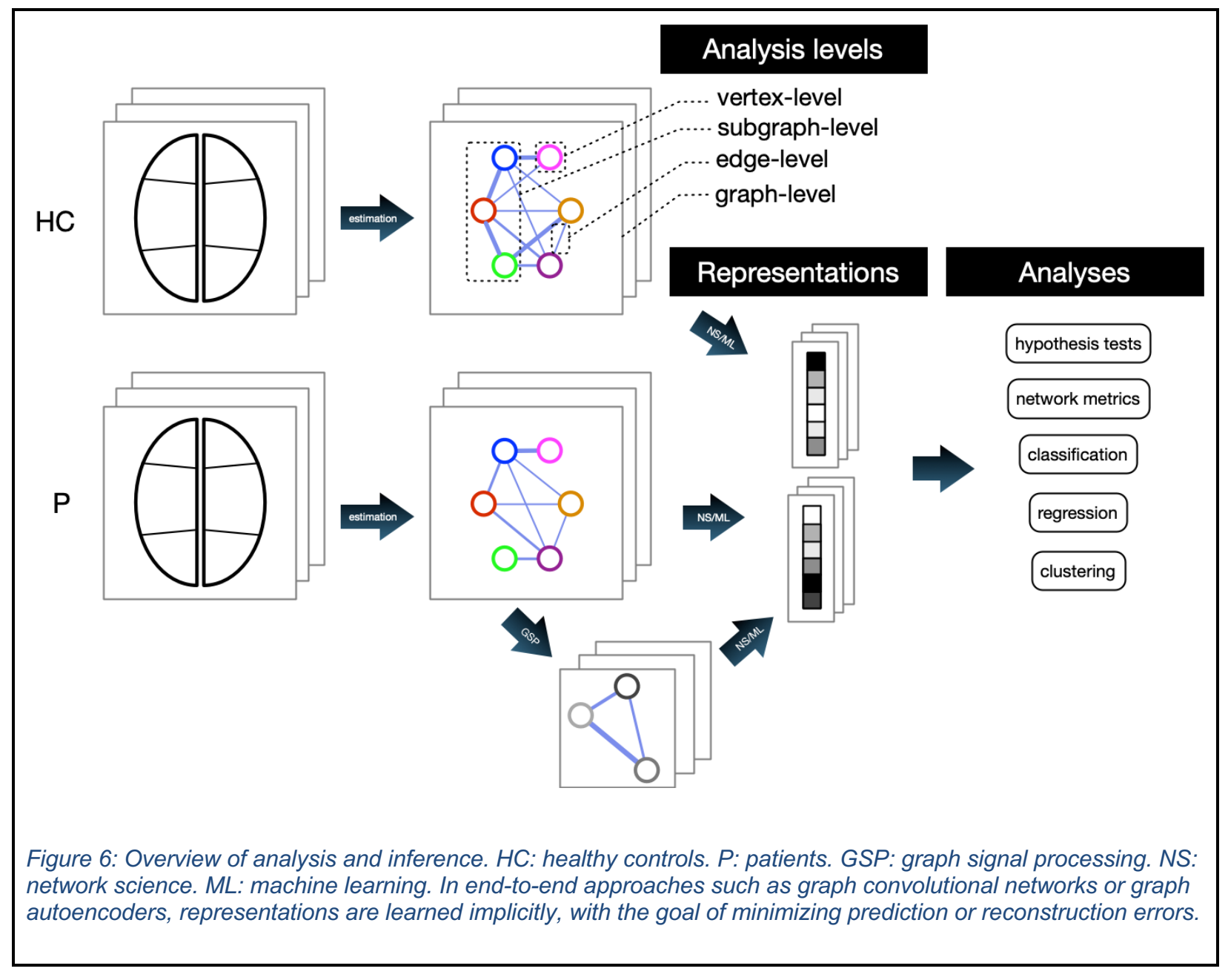


References:

Argyelan, M., Lencz, T., Kaliora, S., Sarpal, D.K., Weissman, N., Kingsley, P.B., Malhotra, A.K., Petrides, G., 2016. Subgenual cingulate cortical activity predicts the efficacy of electroconvulsive therapy. Transl Psychiatry 6, e789. https://doi.org/10.1038/tp.2016.54

Caballero-Gaudes, C., Reynolds, R.C., 2017. Methods for cleaning the BOLD fMRI signal. Neurolmage, Cleaning up the fMRI time series: Mitigating noise with advanced acquisition and correction strategies 154, 128-149. https://doi.org/10.1016/j.neuroimage.2016.12.018

Caeyenberghs, K., Metzler-Baddeley, C., Foley, S., Jones, D.K., 2016. Dynamics of the Human Structural Connectome Underlying Working Memory Training. J. Neurosci. 36, 40564066. https://doi.org/10.1523/JNEUROSCI.1973-15.2016

Cao, H., Zhou, H., Cannon, T.D., 2020. Functional connectome-wide associations of schizophrenia polygenic risk. Mol Psychiatry. https://doi.org/10.1038/s41380-020-0699-3

Carrera, E., Tononi, G., 2014. Diaschisis: past, present, future. Brain 137, 2408-2422. https://doi.org/10.1093/brain/awu101

Cole, J.H., Franke, K., 2017. Predicting Age Using Neuroimaging: Innovative Brain Ageing Biomarkers. Trends in Neurosciences 40, 681-690. https://doi.org/10.1016/j.tins.2017.10.001

Damoiseaux, J.S., 2017. Effects of aging on functional and structural brain connectivity. Neurolmage 160, 32-40. https://doi.org/10.1016/j.neuroimage.2017.01.077

Damoiseaux, J.S., Seeley, W.W., Zhou, J., Shirer, W.R., Coppola, G., Karydas, A., Rosen, H.J., Miller, B.L., Kramer, J.H., Greicius, M.D., 2012. Gender Modulates the APOE $\varepsilon 4$ Effect in Healthy Older Adults: Convergent Evidence from Functional Brain Connectivity and Spinal Fluid Tau Levels. J. Neurosci. 32, 8254-8262. https://doi.org/10.1523/JNEUROSCI.0305-12.2012

Faivre, A., Robinet, E., Guye, M., Rousseau, C., Maarouf, A., Le Troter, A., Zaaraoui, W., Rico, A., Crespy, L., Soulier, E., Confort-Gouny, S., Pelletier, J., Achard, S., Ranjeva, J.-P., Audoin, B., 2016. Depletion of brain functional connectivity enhancement leads to disability progression in multiple sclerosis: A longitudinal resting-state fMRI study. Mult Scler 22, 1695-1708. https://doi.org/10.1177/1352458516628657

Farb, N.A.S., Grady, C.L., Strother, S., Tang-Wai, D.F., Masellis, M., Black, S., Freedman, M., Pollock, B.G., Campbell, K.L., Hasher, L., Chow, T.W., 2013. Abnormal network connectivity in frontotemporal dementia: evidence for prefrontal isolation. Cortex 49, 1856-1873. https://doi.org/10.1016/j.cortex.2012.09.008

Ge, J., Peng, G., Lyu, B., Wang, Y., Zhuo, Y., Niu, Z., Tan, L.H., Leff, A.P., Gao, J.-H., 2015. Cross-language differences in the brain network subserving intelligible speech. Proc Natl Acad Sci U S A 112, 2972-2977. https://doi.org/10.1073/pnas.1416000112

Harricharan, S., Rabellino, D., Frewen, P.A., Densmore, M., Théberge, J., McKinnon, M.C., Schore, A.N., Lanius, R.A., 2016. fMRI functional connectivity of the periaqueductal gray in PTSD and its dissociative subtype. Brain Behav 6, e00579. https://doi.org/10.1002/brb3.579

Horn, A., Reich, M., Vorwerk, J., Li, N., Wenzel, G., Fang, Q., Schmitz-Hübsch, T., Nickl, R., Kupsch, A., Volkmann, J., Kühn, A.A., Fox, M.D., 2017. Connectivity Predicts deep brain stimulation outcome in Parkinson disease. Ann Neurol 82, 67-78. https://doi.org/10.1002/ana.24974

Jones, D.K., Knösche, T.R., Turner, R., 2013. White matter integrity, fiber count, and other fallacies: The do's and don'ts of diffusion MRI. Neurolmage 73, 239-254. https://doi.org/10.1016/j.neuroimage.2012.06.081

Liu, Y., Wang, H., Duan, Y., Huang, J., Ren, Z., Ye, J., Dong, H., Shi, F., Li, K., Wang, J., 2017. Functional Brain Network Alterations in Clinically Isolated Syndrome and Multiple 
Sclerosis: A Graph-based Connectome Study. Radiology 282, 534-541.

https://doi.org/10.1148/radiol.2016152843

Meskaldji, D.-E., Vasung, L., Romascano, D., Thiran, J.-P., Hagmann, P., Morgenthaler, S., Van De Ville, D., 2015. Improved statistical evaluation of group differences in connectomes by screening-filtering strategy with application to study maturation of brain connections between childhood and adolescence. Neurolmage 108, 251-264. https://doi.org/10.1016/j.neuroimage.2014.11.059

Nomi, J.S., Vij, S.G., Dajani, D.R., Steimke, R., Damaraju, E., Rachakonda, S., Calhoun, V.D., Uddin, L.Q., 2017. Chronnectomic patterns and neural flexibility underlie executive function. Neurolmage 147, 861-871. https://doi.org/10.1016/j.neuroimage.2016.10.026

Sadaghiani, S., Poline, J.-B., Kleinschmidt, A., D’Esposito, M., 2015. Ongoing dynamics in large-scale functional connectivity predict perception. Proc Natl Acad Sci USA 112, 8463-8468. https://doi.org/10.1073/pnas.1420687112

Sair, H.I., Yahyavi-Firouz-Abadi, N., Calhoun, V.D., Airan, R.D., Agarwal, S., Intrapiromkul, J., Choe, A.S., Gujar, S.K., Caffo, B., Lindquist, M.A., Pillai, J.J., 2016. Presurgical brain mapping of the language network in patients with brain tumors using resting-state fMRI: Comparison with task fMRI. Hum Brain Mapp 37, 913-923. https://doi.org/10.1002/hbm.23075

Schlemm, E., Schulz, R., Bönstrup, M., Krawinkel, L., Fiehler, J., Gerloff, C., Thomalla, G., Cheng, B., 2020. Structural brain networks and functional motor outcome after stroke-a prospective cohort study. Brain Communications 2. https://doi.org/10.1093/braincomms/fcaa001

Scott-Van Zeeland, A.A., Abrahams, B.S., Alvarez-Retuerto, A.I., Sonnenblick, L.I., Rudie, J.D., Ghahremani, D., Mumford, J.A., Poldrack, R.A., Dapretto, M., Geschwind, D.H., Bookheimer, S.Y., 2010. Altered functional connectivity in frontal lobe circuits is associated with variation in the autism risk gene CNTNAP2. Sci Transl Med 2, 56ra80. https://doi.org/10.1126/scitranslmed.3001344

Seeley, W.W., Crawford, R.K., Zhou, J., Miller, B.L., Greicius, M.D., 2009. Neurodegenerative diseases target large-scale human brain networks. Neuron 62, 42-52. https://doi.org/10.1016/j.neuron.2009.03.024

Shen, X., Finn, E.S., Scheinost, D., Rosenberg, M.D., Chun, M.M., Papademetris, X., Constable, R.T., 2017. Using connectome-based predictive modeling to predict individual behavior from brain connectivity. Nat Protoc 12, 506-518. https://doi.org/10.1038/nprot.2016.178

Shine, J.M., Bissett, P.G., Bell, P.T., Koyejo, O., Balsters, J.H., Gorgolewski, K.J., Moodie, C.A., Poldrack, R.A., 2016. The Dynamics of Functional Brain Networks: Integrated Network States during Cognitive Task Performance. Neuron 92, 544-554. https://doi.org/10.1016/j.neuron.2016.09.018

Spoormaker, V.I., Schröter, M.S., Gleiser, P.M., Andrade, K.C., Dresler, M., Wehrle, R., Sämann, P.G., Czisch, M., 2010. Development of a large-scale functional brain network during human non-rapid eye movement sleep. J Neurosci 30, 11379-11387. https://doi.org/10.1523/JNEUROSCI.2015-10.2010

Tax, C.MW., Grussu, F., Kaden, E., Ning, L., Rudrapatna, U., John Evans, C., St-Jean, S., Leemans, A., Koppers, S., Merhof, D., Ghosh, A., Tanno, R., Alexander, D.C., Zappalà, S., Charron, C., Kusmia, S., Linden, D.EJ., Jones, D.K., Veraart, J., 2019. Crossscanner and cross-protocol diffusion MRI data harmonisation: A benchmark database and evaluation of algorithms. Neurolmage 195, 285-299. https://doi.org/10.1016/j.neuroimage.2019.01.077

Zalesky, A., Fornito, A., Bullmore, E.T., 2010. Network-based statistic: identifying differences in brain networks. Neuroimage 53, 1197-1207. https://doi.org/10.1016/j.neuroimage.2010.06.041 